\title{
Théâtre des disparues : Le Premier Jardin
}

ANDRÉA KING

UNIVERSITÉ PuRDUE

Résumé. Cette étude du Premier Jardin explore le legs historique et scénique au féminin que s'engage à mettre en lumière Flora, le personnage principal du roman. Flora, qui est comédienne, revendique une création au féminin sans pour autant échapper à un système patriarcal qui dépend de l'effacement du féminin. Cette revendication ambivalente et problématique se déploie par le palimpseste : les divers rôles qu'endosse Flora relèvent de l'Histoire et du récit théâtral.

Mots-clés : Femmes, Féminin, Création, Théâtre, Histoire, Palimpseste, Intertextualité, Spectres

Le Premier Jardin d'Anne Hébert présente la mise en scène d'un legs historique et scénique au féminin dont hérite le personnage principal du roman, Flora Fontanges, legs qui s'exprime dans le texte par des palimpsestes oraux (légendes des femmes d'antan qui ont habité la ville de Québec) ou écrits (intertextes théâtraux). En effet, Flora, qui est comédienne et mère, exploite les histoires de femmes « réelles » (filles du Roi, servantes, orphelines, épouses involontaires) et théâtrales (personnages féminins) afin de mettre en lumière l'effacement du féminin sur les plans esthétique et socio-historique. En nous servant de théories et de critiques féministes, nous ferons une analyse du théâtre pour lequel s'engage Flora, soulignant ainsi les nuances genrées du projet de création - de reconstitution et de revendication - qu'entreprend celle-ci. Nous montrerons que les usages du palimpseste du roman participent à une remise en question ambivalente de divers aspects de la féminité telle que cette dernière est socialement construite : maternité, passivité, effacement de soi devant l'autre.

Retour de l'Histoire

Dans I'incipit du Premier Jardin, Flora revient dans sa ville natale (la lectrice comprend qu'il s'agit de Québec) pour jouer Winnie dans une mise en scène d'Oh les beaux jours de Samuel Beckett. Elle apprend de Raphaël, le copain de sa fille, Maud, 
que celle-ci a disparu. Flora est hantée par les femmes qui ont habité cette ville ancienne, à savoir toutes celles dont la vie et la mort constituent les fondations de la culture canadienne-française. Flora revit les souffrances genrées de ces femmes en étudiant I'histoire de la ville. Raphaël, qui est guide touristique, s'avère l'inspiration de ces jeux d'apparition et de disparition historiques. Flora et Raphaël se promènent en ville, « interprétant » tous deux la vie d'« obscures héroïnes de l'histoire » québécoise. (Le Premier Jardin, 1988 : 120) Raphaël fournit des détails historiques; Flora joue le rôle des divers personnages. Il faudrait noter que la plupart des « héroïnes » qu'interprètent Flora et Raphaël le sont malgré elles : à part le personnage de Marie Rollet, mère mythique du peuple canadien-français, aucune de ces femmes d'antan (à tout le moins selon la représentation historique hébertienne) n'a choisi son propre destin. Les corps de ces femmes constituaient néanmoins la pierre angulaire du Québec. Si Flora s'identifie à ces femmes, c'est qu'elle reconnaît sa dette personnelle envers elles : elle n'est qu'un maillon dans « une longue chaîne de vie, commencée il $y$ a trois siècles $\gg .\left(P J^{1}: 100\right)$

Comme le note Anne Ancrenat, «plus que dans les romans précédents [d'Anne Hébert], la transmission de I'histoire des femmes sera présente [dans Le Premier Jardin] et son rôle efficient puisque c'est I'histoire collective qui aidera à l'émergence de l'histoire privée. » (Ancrenat, 2002 : 195) Flora Fontanges ne peut comprendre son passé en tant qu'orpheline, et son présent en tant que femme, sans tracer une filiation féminine historique. Selon Lori Saint-Martin, cette mise en texte de la filiation dans le roman s'avère inéluctablement liée à la création (littéraire) au féminin : « Tout se produit comme si, pour une femme qui écrit, toute tentative de repenser I'Histoire officielle ou l'histoire familiale passait forcément par l'intégration du féminin, et particulièrement du maternel, refoulé. » (Saint-Martin, 2006 : 210)

Parmi les héroïnes historiques qui forment cette «longue chaîne de vie », une grande place est réservée aux filles du Roi. Hébert met en relief la peur et la souffrance qui auraient marqué ces premières femmes coloniales et colonisées, arrachées de la Salpêtrière, traînées dans des bateaux destinés à la Nouvelle-France, et mariées par la suite à des inconnus. Ces femmes sont à la fois sujets et objets de la hantise : « Trente passagers sont morts en cours de route, et il a fallu les jeter à la mer comme des pierres. Les survivantes encore longtemps seront hantées par le roulis et le tangage, tant il est vrai que ce grand brassement de l'océan habite toujours leurs 
corps, de la racine des cheveux à la pointe des orteils. » ( $P J: 97)$ Flora se vêtira de cette histoire (conjurée par Raphaël) comme d'un collier couleur de mer, et là où il y avait de la pierre croîtra la filiation féminine : « [Flora et Raphaël] ont appelé des créatures disparues, les tirant par leur nom, comme avec une corde du fond d'un puits ». ( $P J: 120)$ L'énumération des noms des femmes disparues,

Graton, Mathurine

Gruau, Jeanne

Guerrière, Marie-Bonne

Hallier, Perette

d'Orange, Barbe (PJ : 99),

souligne de façon visuelle ces enjeux associatifs fantomatiques et fantasmatiques. Par la représentation typographique verticale, le fil passe d'une femme à l'autre avant d'être saisi et approprié par Flora, qui, elle, incarne à merveille la juxtaposition pierre/fil. À I'orphelinat, on I'avait baptisée Pierrette Paul; elle est devenue la stoïque Marie Éventurel par la suite avant d'enfiler le nom plus mouvant de Flora Fontanges, désignation qui marque l'état de créativité autonome qui la caractérise dans le présent de la narration.

L'onomastique est au premier chef en ce qui concerne la valorisation du féminin dans le roman, comme le montrent Ancrenat et Saint-Martin. Pour Ancrenat, le passage du nom Pierrette Paul (P. P.) à celui de Flora Fontanges (F. F.) connote les termes de musique pianissimo et fortissimo, lesquels « schématisent le parcours [du personnage] comme un cheminement qui va crescendo. » (Ancrenat, 2002 : 195) Lori Saint-Martin note que «[n]ommer, c'est créer, rien de moins », et, en citant l'exemple des bonnes d'antan - qui figurent de façon importante dans la reconstitution historique de Flora -, elle constate qu'« [i]nversement, la perte du nom propre est liée à une perte d'identité qui conduit à la mort » (Saint-Martin, 2006 : 216). En effet, Hébert insiste pour que ces femmes d'antan « viennent saluer sur la scène et se nommer bien haut, afin qu'on les reconnaisse et leur rende hommage, avant qu'elles ne disparaissent à nouveau. » $(P J: 120)$ Or, elle les lie et «les ram[ène] sur le rivage, dans leurs cendres légères » $(P J: 103)$, comme pour mettre en lumière la douce force qui les caractérisait. 


\section{Filiations scéniques}

Une discussion de la filiation scénique fournit une occasion d'éclaircir un aspect intertextuel du Premier Jardin qui a été négligé par la critique jusqu'à présent, à savoir l'importance et la signification des divers rôles théâtraux que joue Flora.

Bon nombre des personnages théâtraux qu'interprète Flora meurent victimes de l'amour, compris ici dans le sens idéologique patriarcal du terme, idéologie qui construit la femme comme objet d'échange entre hommes. Ces personnages sont : Adrienne Lecouvreur, Jeanne d'Arc, Phèdre, Hedda Gabler, Mlle Julie et Ophélie². Quatre de ces personnages se suicident en raison d'une peine d'amour romantique (Phèdre, Hedda, Mlle Julie, Ophélie); I'un se sacrifie pour I'amour du Père (Jeanne); l'autre est assassiné par une amante rivale (Adrienne). L'exception à la règle est le personnage de Fantine, dont le décès est lié à la peine d'amour maternel. Flora donne une nouvelle vie à ces personnages féminins, mais force est de noter que ceux-ci lui donnent une nouvelle mort chaque fois qu'elle les interprète. À la fois créatrice et médium, Flora s'efface pour donner corps aux gestes et aux paroles d'une autre, ne ressurgissant qu'au moment de la disparition de celle-ci. À travers elle, l'amour patriarcal est continuellement mis en scène et démonté; incarné et décomposé.

Certains de ces personnages théâtraux s'avèrent être des moyens par lesquels Flora s'approche des compatriotes historiques susmentionnées. Ophélie - le premier personnage qu'interprète Flora - est un de ces rôles. Le souvenir de cette interprétation permet à Flora de mieux comprendre Renée Chauvreux, fille du Roi retrouvée «morte dans la neige » $(P J: 104)$ peu après son arrivée en Nouvelle-France en 1670. En faisant le deuil de Renée, Flora pose à Ophélie la question « de la destinée amère des filles. Pourquoi? » (PJ : 105); Ophélie, dans son silence de noyée, ne saurait répondre.

Le dédoublement scénique et identificatoire se complexifie dans le cas du rôle de Phèdre, car l'interprétation par Flora de ce personnage entraîne Maud dans le théâtre mortifère. C'est à l'époque où Flora est enceinte de Maud et abandonnée par le père de celle-ci qu'elle joue ce rôle; or, elle « porte sa fille comme le fruit de deux amours » (PJ : 109), I'un disparu, l'autre inventé. Il s'agit, bien entendu, de l'amour de I'homme absent, et celui du rôle de Phèdre lui-même (ou des rôles de théâtre en général). Ainsi, même avant sa naissance - a fortiori bien avant sa disparition -,

2. Winnie ne meurt pas à la fin de la pièce, mais l'on comprend qu'elle sera enterrée vivante dans le mamelon de terre où elle se retrouve. Nous y reviendrons. 
Maud incarne ce qui est absent, ce qui s'envole, voire ce qui est mort. Car, en effet, c'est comme si elle était vouée d'avance à un redoublement mortifère. En tant que fœtus - et à l'instar des personnages de théâtre qui « empruntent » le corps de Flora -, Maud phagocyte la vie de sa mère ${ }^{3}$, au point où celle-ci faillit mourir lors de sa naissance $(P J: 109)$. Dans la mesure où elle joue le rôle de Phèdre par procuration - à savoir depuis la matrice -, Maud est aussi impliquée dans le théâtre mortifère que sa mère.

Ce redoublement représentatif fournit justement une première explication aux disparitions de Maud dans son adolescence tout autant qu'à l'âge adulte, disparitions qui correspondent toujours à l'endossement par Flora d'un nouveau rôle ( $P J: 62$ ). En s'effaçant, la fille renouerait avec le premier lien spectral avec la mère : elle disparaît pour mieux participer au théâtre de l'autre.

Winnie, le miroir et le mamelon

Examinons maintenant le rôle central de ce théâtre de l'autre, celui de Winnie dans Oh les beaux jours. Un éclairage brutal caractérise la pièce de Beckett; cet éclairage est hautement symbolique, et Hébert l'exploite pour créer une cohérence thématique dans son roman. En effet, puisque les autres personnages qu'interprète Flora naissent, de diverses façons, « en pleine lumière » $(P J: 62)$, ils sont tous des précurseurs de Winnie ${ }^{4}$. Une exploration de ce personnage et du symbolisme auquel il prend part mènera donc à une compréhension plus profonde de l'intertextualité hébertienne.

Le soleil brûle sans arrêt sur Winnie d'Oh les beaux jours, comme si son existence constituait une seule journée interminable et affreuse. Affreuse, car Winnie est enterrée jusqu'à la poitrine dans un mamelon de terre au début de la pièce, et qu'elle n'aura pas la possibilité de s'en sortir. Loin de là, en fait : elle y sera ensevelie jusqu'au cou au commencement du deuxième acte. Son mari - qui s'appelle Willie - habite dans un trou derrière le mamelon de terre. Puisque Winnie ne peut bouger, elle se contente de parler - à elle-même et à Willie, qui ne répond presque jamais - et de jouer avec les quelques objets à sa disposition : miroir, brosse à dents, lunettes, parasol brisé, etc. Tout au long de la pièce, elle s'efforce de garder une attitude opti-

3. Cette phagocytose créative/fœtale est d'ailleurs explicitée à l'incipit du roman, où Flora est « grosse de la petite figure fripée de Winnie »d'Oh les beaux jours $(P J: 11)$.

4. Par exemple, le rôle d'Ophélie se joue « sur une scène violemment éclairée » $(P J: 105)$, et celui de Phèdre s'alimente de la «fureur de flamme ravageuse » ( $P J: 109)$ de l'amante abandonnée. 
miste à l'égard de sa situation abominable.

Comme le font remarquer plusieurs critiques beckettiennes féministes, les difficultés de Winnie, et sa réaction face à celles-ci, s'avèrent incontestablement genrées. « Conditionnée pour "tirer le meilleur parti des choses" $[\ldots]$, comme reconstruire l'apparence physique de quelqu'un, Winnie en passe par les rituels que la société assigne, et permet, aux femmes », observe par exemple Linda Ben-Zvi (1990 : xii; nous traduisons). Il importe de noter qu'il ne s'agit pas d'une représentation misogyne, cependant, car, comme le souligne Shari Benstock, cette pièce de Beckett remet en question les valeurs qu'elle met en scène, celles qui fixent la femme « à sa place », à savoir celles qui l'emprisonnent comme objet du désir masculin (Benstock, 1990 : 183). Cette remise en question se fait depuis l'intérieur de la structure patriarcale elle-même :

[Winnie] prend la parole depuis le système culturel qu'elle interroge, depuis le monticule qui représente non pas la « mère nature » à partir de termes traditionnels relevant du motif de la fécondité, mais plutôt la boue, le détritus d'une civilisation qui, à la fois idolâtre, et ensevelit les femmes.

Il n'y a pas de lieu pour elle en dehors du système, au-delà du regard patriarcal, à partir duquel remettre en question le système, et c'est cette « vérité » que Oh les beaux jours révèle. [...] La place des femmes se trouve à l'endroit du monticule, utérus débordant, sein fait d'argile, lieu d'enterrement [...] Winnie doit être ancrée au sol parce que c'est précisément sa fixité qui permet au système d'évoluer sans accroc. (Benstock, 1990 : 183; nous traduisons)

Anne Hébert, à travers le palimpseste, joue sur cet ensevelissement de la femme dans une économie masculine destructrice : le roman met en scène la mise en scène de ce type d'enterrement social.

Le personnage du metteur en scène d'Oh les beaux jours est une figure clé en ce qui concerne le discours critique palimpsestique déployé dans le roman. Comme pour maîtriser la créativité au féminin, il interdit à Flora de sourire lors des répétitions ( $P J: 27$ ), et lui demande aussi de faire un régime, car « [i]l la désire cassante comme du verre, grièvement blessée, entièrement soumise à la vieillesse et à la mort. » (PJ : 69) Il l'isole du comédien qui joue le rôle de Willie, tout autant que de l'équipe technique, créant ainsi « $[u]$ ne sorte de huis clos » qui lui permettrait de la regarder « comme avec une loupe » - tel un « microbe »- et de « se délect[er] intensément du spectacle » qu'il crée ( $P J: 45-46)$. Le regard perçant de ce personnage, symbolisé par les lunettes dorées qu'il porte durant les répétitions (et qu'il ajuste à plusieurs reprises), met l'accent sur le positionnement privilégié que tien- 
drait l'homme dans le processus créatif, et sur le fait que ce soit sa conception du monde qui s'en trouve relayée. En effet, les féministes n'ont-elles pas montré que la définition traditionnelle du génie est forcément masculine ${ }^{5}$ ? Les lunettes du metteur en scène rappellent, par ailleurs, celles que manie Winnie dans la pièce de Beckett, mais qu'elle choisit de remettre dans son sac à main en disant qu'elle a déjà « assez vu ». (Beckett, $1974: 15)$ Que les lunettes changent de mains (ou de visage), cette transposition souligne I'origine masculine du regard.

Winnie a peut-être « assez vu», mais le personnage masculin témoigne d'une « [i]nsistance forcenée de myope » $(P J: 69)$, et il « attendra le temps qu'il faut pour que la déchéance de Flora Fontanges s'accomplisse » $(P J: 69)$ devant ses yeux. Autrement dit, il attendra la disparition de la femme - l'incarnation parfaite de celle-ci dans son rôle de morte, « [l]'ensablement d'une créature vivante [...], grain à grain, soir après soir, au théâtre d'été de I'Emérillon, tel qu['il] le rêve » $(P J: 14)^{6}-$, ce qui lui permettra d'affirmer son propre génie.

Flora témoigne d'un malaise vis-à-vis de ce rôle spéculaire - celui de la femme « à sa place »- dans lequel le personnage du metteur en scène voudrait l'ensevelir. Mais, curieusement, au moment où cet homme la scrute, elle ne peut s'empêcher de conjurer l'image de sa fille, comme pour enterrer Maud dans le mamelon à son tour. Cet ensevelissement fantasmatique surprend Flora : «Quelle idée de m'amener ma fille à un moment pareil, dit-elle. [...] Je suis vidée, morte... » $(P]: 47)$ Flora énoncera deux fois de plus cette dernière phrase dans une sorte de répétition compulsionnelle ( $P J$ : 47-49). Lors de la deuxième instance, la figure de Maud s'effacera « pour faire place à la silhouette voûtée du directeur de l'Emérillon » ( $P J: 47)$; la troisième donnera lieu, une fois de plus, à l'apparition filiale. Se forme donc un triangle qui lie mère et fille, mais qui passe inéluctablement par l'angle mort qu'est le regard esthétisant de I'homme.

On lit dans le même passage : «Le visage de [l]a fille choisit pour apparaître ce moment précis où Flora Fontanges, sans défense, semble s'extraire à grand-peine $d^{\prime}$ un tas de bois mort. » ( $P J: 47$; nous soulignons) Grâce à cette assimilation du mamelon de terre de Winnie à du bois, les associations génésiaques du roman - déployées par le leitmotiv de l'arbre - ne peuvent se lire qu'en rapport avec l'ensevelissement de la fille. Monique Boucher-Marchand soutient que l'arbre est un des sym-

5. Voir par exemple Christine Battersby.

6. Le metteur en scène et le « directeur » auquel fait référence ce passage sont une seule et même personne. 
boles par lesquels Hébert «bouleverse la lecture figée de l'histoire » et contribue à la création d'une « lignée matrilinéaire » qui commencerait avec la première femme (Boucher-Marchand, $2000: 51)^{7}$. Une telle lecture est d'ailleurs confortée par le poème « Ève » d'Anne Hébert, qui, d'une certaine façon, sert de garant autotextuel pour toute interprétation du roman qui mettrait en valeur le féminin ${ }^{8}$ : «Mère du Christ souviens-toi des filles dernières-nées, de celles qui sont sans nom ni histoire, tout de suite fracassées entre deux très grandes pierres / [...] Que ta mémoire se brise au soleil, et, au risque de réveiller le crime endormi, retrouve l'ombre de la grâce sur ta face comme un rayon noir. » (Fuvre poétique, 1993 : 88-90) Mais le symbole de l'arbre/du bois est extrêmement complexe : I'arbre vivant représente le principe vital, la fécondité féminine; le « bois mort » évoque l'anéantissement de la femme. Le « bois mort » est associé à Rosa Gaudrault, la seule «mère » que Flora ait connue et qui a péri dans l'incendie à l'orphelinat. On retrouve Rosa «carbonisé[e] », deux petites filles dans les bras, toutes trois « couvertes de glace, en une seule branche noire, tordue. » ( $P J: 129)$ Le bois mort représente aussi Flora elle-même, qui, à l'encontre de la figure mythique du phénix, n'est pas sortie triomphante de cette mort psychique, mais plutôt «muette et glacée, comme pétrifiée. » ( $P J: 132)$ Cet événement traumatisant donnera lieu à des cauchemars affreux; les cris nocturnes que pousse le personnage lors de ses premières nuits chez les Éventurel en témoignent : « Du bois mort, là, là! » ( $P J: 129)$, hurle la jeune Flora, nommée Marie à cette époque-là de sa vie.

Nous l'avons déjà dit : par des jeux de rôles historiques, Flora fait vivre des femmes tues, disparues, et oubliées. Mais, paradoxalement, elle ne peut s'empêcher d'engluer sa fille «fugueuse » - qui est bel et bien vivante - dans un passé révolu et meurtrier. Elle l'amène sous la lumière crue de la scène d'Oh les beaux jours, lieu d'une brûlure représentative, personnelle et historique. Cette lumière ne nourrit pas, elle tue : comme l'observe Lucille Roy dans sa discussion du « Tombeau des rois », elle « a une puissance captivante, écrasante et néfaste. Son intensité dessèche; et son poids, effet apparent de sa densité, pousse graduellement l'être dans l'abîme. » (Roy,

7. Boucher-Marchand s'appuie sur deux passages en particulier. L'un évoque la première femme québécoise, Marie Rollet (épouse de Louis Hébert), mais en I'occurrence « fragmentée en mille frais visages » ( $P J: 99)$, c'est-à-dire complexifiée, hétérogénéisée : « Des branches vertes lui sortent d'entre les cuisses, c'est un arbre entier, $[\ldots]$, et nous sommes au monde comme des enfants étonnés » $(P J: 100)$. L'autre présente le fantasme du personnage féminin (nommé Marie Éventurel à ce moment-là dans le récit) à l'occasion de sa sortie de l'orphelinat : « Depuis toujours, elle est sans racines et rêve d'un grand arbre, ancré dans la nuit de la terre, sous la ville, soulevant l'asphalte des trottoirs et des rues, rien qu'avec le souffle noir de son haleine souterraine. » $(P J: 124)$

8. Outre Boucher-Marchand, voir Lori Saint-Martin (2006) et Anne Fonteneau (2000). 
2006 : 54) Il faut noter que Maud est assimilée à ce champ de lumière spectralisante même avant que ne débutent les répétitions de la pièce de Beckett : en décrivant sa première rencontre avec Maud à l'aéroport de Lorette, Raphaël compare la jeune femme à un «fantôme » $(P J: 59)$, fantôme qui lui « a demandé du feu. » ( $P J: 59)$

\section{Agentivité, spectralité et intertextualité}

Flora est une actrice réputée, une femme qui gagne bien sa vie, et qui est fière de dire que sa fille porte son nom et non celui d'un homme ( $P J$ : 109). Mais il y a des limites à son agentivité : loin de choisir le rôle de femme ensevelie, Flora est « convoquée » $(P J: 10)$ pour le jouer ${ }^{9}$, « tirée de sa retraite $[\ldots]$ comme une plante que I'on sort de l'ombre et ramène vers le jour ». ( $P J: 35)$ Nous l'avons constaté : par l'entremise du rôle de Winnie, Flora se retrouve pétrifiée dans et par le regard masculin, aux prises avec un passé qu'elle n'arrivera jamais tout à fait à assimiler. Et tout comme Winnie, elle aura recours à l'optimisme face à ce rôle. « C'est si beau de jouer ! $(P J: 47)$, dit-elle en répétitions, lorsque la figure de sa fille s'est atténuée, et que le metteur en scène cesse de la scruter. De telles formules beckettiennes reviennent aux moments difficiles de la remémoration de Flora, mais avec une ironie croissante. Par exemple, à la suite de l'abandon de Flora par le père de Maud, on lit : «C'est le beau temps. » (PJ : 108) Ou encore :

Salut, sainte lumière!

Amertume et dérision [...]. La plus profonde solitude vient vers elle, émergeant à peine du grand beau temps qu'il fait.

Oh! le beau jour encore que ça va être! (PJ : 185)

après que Flora a pris connaissance de la réconciliation de Maud et de Raphaël.

Les retrouvailles du jeune couple sont donc marquées d'une ambiguïté qui incite à une exploration plus poussée. Tout comme le symbole de l'arbre ne peut se lire qu'en concordance avec celui du mamelon de terre beckettien, la réconciliation du jeune couple doit se lire en lien avec une autre référence intertextuelle : I'allusion à la fin du

9. Paul Côté et Constantina Mitchell décèlent une autre ironie dans le rôle que joue Flora, à savoir le fait que cette dernière semble témoigner d'un désir inconscient de se soumettre à la « fausse grandmère » cruelle de sa jeunesse, la grand-mère Éventurel : « Le tronçonnement visuel de Winnie, "enterrée jusqu'au cou",[...] semble une réalisation théâtrale de la décapitation symbolique par quoi la fausse grand-mère proscrivait la jeune Flora : "La petite fille a très bien compris que la Reine de Cœur la condamnait à avoir la tête tranchée". » (Côté et Mitchell, 1991 : 457) 
roman Chacun sa vérité de Luigi Pirandello ${ }^{10}$. Car le dénouement du récit laisse entendre que le prochain rôle que jouera Flora est celui de Mme Frola dans cette pièce qui, tout comme le roman d'Anne Hébert, figure des couplements étranges et des retrouvailles ambiguës. Afin de faire ressortir les importants liens thématiques entre les deux textes, nous ferons ici un court résumé de la pièce.

Chacun sa vérité remet en question la notion d'objectivité. Les habitants d'un village italien sont curieux vis-à-vis des nouveaux arrivants, le fonctionnaire M. Ponza et sa femme, et la mère de celle-ci, Mme Frola. Le couple habite en appartement, mais, à l'encontre des mœurs, M. Ponza a aussi loué pour sa belle-mère un logement à l'autre bout du village. On ne voit jamais l'épouse, mais on observe que I'homme rend régulièrement visite à la vieille dame. Les questions abondent : I'homme a-t-il enfermé sa femme (ou bien sa belle-mère) à la maison? Ou bien les deux femmes ont-elles choisi cet arrangement? Ces gens cachent-ils un passé malsain? Appelés par les villageois à s'expliquer, le fonctionnaire et la belle-mère fournissent à tour de rôle plusieurs versions contradictoires de leur situation.

Selon la première explication de Mme Frola - elle en fournira deux au cours de la pièce -, elle aurait accepté cet enfermement parce que son beau-fils était jaloux mais très aimant, et qu'elle ne voulait pas compromettre le bonheur de sa fille en violant les consignes du mari. L'explication du beau-fils s'oppose cependant à cette histoire : il « révèle » que sa belle-mère est folle, que sa femme est morte quatre ans auparavant lors d'un tremblement de terre, et que la vieille dame fut ensuite internée dans un asile parce qu'elle refusait d'y croire. Selon cette version des événements, $M$. Ponza se serait remarié, et la belle-mère se serait convaincue que la nouvelle femme était sa fille. D'après $M$. Ponza, depuis la sortie de l'asile de la belle-mère, le couple joue une sorte de comédie qui vise à protéger la vieille dame. La nouvelle épouse resterait chez elle pour éviter de jouer un rôle de revenante qui la rend mal à l'aise.

La deuxième version de I'histoire de Mme Frola «corrige » encore une fois l'information fournie par le beau-fils. Ce n'est pas elle, mais sa fille qui aurait passé du temps dans un asile : puisque Mme Ponza ne savait répondre à la passion trop exigeante de son mari, les médecins lui auraient ordonné du repos et de la solitude.

10. Lori Saint-Martin soulève aussi cette référence, parlant de son importance pour la compréhension du rapport mère-fille dans Le Premier Jardin : « La pièce [...] met en scène une mère séparée de sa fille pour des raisons obscures liées au mari de celle-ci [...], tout comme Raphaël sépare finalement Flora et Maud. La presque coïncidence des noms de l'actrice au rôle - Flora, Frola - n'est pas fortuite : elle révèle au contraire que les moindres détails du roman prennent tout leur sens à la lumière du rapport mère-fille. » (Saint-Martin, 2006 : 215) 
Selon cette version des événements, le mari croyait sa femme morte et serait devenu fou. Lors de la sortie de l'asile de la fille, cette dernière aurait accepté de participer à de secondes noces et à jouer le rôle de seconde épouse, afin de ne pas déstabiliser son mari. Mme Frola, quant à elle, aurait participé à la ruse par amour maternel.

Il importe de noter que I'on ne saura jamais la «vraie » version des événements : lorsque la femme de $M$. Ponza se présente enfin, elle est émue et étreint Mme Frola, mais elle refuse de céder à la soif de vérité des villageois. À la suite de ces retrouvailles émotives, M. Ponza escorte Mme Frola jusqu'à son logement. Rideau.

Outre certaines dissimilitudes évidentes, les versions de I'histoire présentées ont quatre choses en commun : 1) la séparation douloureuse de la mère d'avec sa fille, séparation qui est remise en scène au moment du dénouement; 2 ) le désir du mari comme obstacle à la relation maternelle; 3 ) la relation privilégiée entre belle-mère et beau-fils; 4) la spectralité de la fille devenue amante. Ce sont tous là des traits que partage Le Premier Jardin. En parlant du roman, Lori Saint-Martin fait remarquer la nécessité de la séparation de Maud et de Flora, qu'elle attribue à leur «similitude », en l'occurrence leur propension à se dérober de la scène et à se fuir l'une l'autre : « Comme si une co-présence véritable des deux femmes était impossible. [...]; infiniment appelées, infiniment différées, les retrouvailles n'occupent qu'une fraction du roman [...], et se soldent par une nouvelle séparation. Signe de l'importance de ce rapprochement comme de son impossibilité. » (Saint-Martin, $2006: 212$ ) Les observations de Saint-Martin pourraient bien s'appliquer à la pièce de Pirandello. Et outre le caractère fuyant des personnages féminins des deux œuvres, ces quatre femmes partagent une seule position symbolique par rapport au personnage masculin, c'està-dire que mères et filles ne peuvent partager la scène créée pour l'homme, par I'homme, et sur laquelle elles ne sont que des comédiennes figées. Bref, il n'y a de place que pour une seule femme à la fois sur la scène patriarcale.

Substitutions et réconciliations risquées

La femme revient toujours de l'abîme, toujours vêtue d'un autre rôle ${ }^{11}$, vouée à jouer le rôle d'une autre. Flora, qui se fait couper les cheveux avant de jouer le rôle de Winnie - qui «s[e] fait la tête de Jeanne au bûcher »-se dit qu'elle «pourrait très bien [ainsi] jouer la passion d'une pucelle de dix-neuf ans que le feu dénude

11. N'oublions pas l'importance de l'aspect vestimentaire en ce qui concerne le rôle de la femme ensevelie. Comme le note Carey Perloff, le mamelon de terre est le costume de Winnie : Beckett crée justement cet « environnement hostile » pour que l'inconfort de l'actrice soit corrélatif à celui du personnage (Perloff, $1994: 166)$. 
avant de la réduire en cendres. » $(P J: 27)$ C'est dire aussi qu'elle pourrait très bien jouer le rôle de remplaçante de sa fille disparue. En effet, Maud n'a-t-elle pas environ le même âge (20 ans) que le personnage de Jeanne d'Arc? Nous avons constaté la phagocytose filiale, mais la passion de la mère ne vampirise-t-elle pas aussi l'existence de la fille? Ce remplacement symbolique de la fille par la mère (auquel participe celle-ci sans en être consciente) fournirait une deuxième explication à la corrélation entre les disparitions de Maud et l'endossement des nouveaux rôles théâtraux de Flora, relation causale qui est notamment mise en représentation par l'intercalation des affiches de spectacle et des notices de disparition sur le mur de l'appartement de Maud et de Raphaël ( $P J$ : 27-28). Car, en effet, Flora se substitue à Maud auprès de Raphaël, du moins en ce qui concerne les désirs de celui-ci. En faisant office de guide dans la ville, Raphaël explique à Flora que leur « plus grand rêve, à Maud et à lui, c'était de vivre une journée entière, sans en perdre un instant. » Il ajoute : « C'est déjà commencé, vous et moi, depuis ce midi. » ( $P J: 43)$ D'ailleurs, à l'avenant de ce dernier commentaire, et après une autre journée passée à conjurer les mortes, Raphaël implore Flora de le « console[r] [...] comme une vraie femme qui ne craint ni Dieu ni diable. » (PJ : 67) Flora repousse cette supplication : la jouissance lui est impossible justement parce qu'elle n'est pas une «vraie femme » dans le scénario qu'imagine Raphaël, mais plutôt une sorte de prosopopée, l'incarnation d'un manque masculin toujours fuyant, mouvant. Mais de quel manque s'agit-il pour Raphaël? Celui de l'amante ou celui de la mère? Des deux, certes. Comme le dit Raphaël à Flora : « Je réveillerai le temps passé. J'en sortirai des personnages encore vivants, enfouis sous les décombres. [...] Vous jouerez tous les rôles de femme ». $(P J: 75)^{12}$

Boucher-Marchand voit dans le redoublement de la mère et de la fille dans Le Premier Jardin un signe de I'ouverture d'une voie féminine positive. Elle soutient $q^{\prime}{ }^{\prime} \ll[e] n$ faisant revivre son passé, [Flora] lui ferme en effet définitivement la porte, pour mieux entrouvrir celle de l'avenir, représentée par sa fille de 20 ans. Symboliquement, mère et fille sont ainsi confondues en un même personnage où la purification de l'une, métaphorique, facilite le passage à la fertilité de l'autre. » (Boucher-Marchand, 2000 : 53) Anne Fonteneau, pour sa part, insiste sur le « métaféminisme » du

12. Ces paroles rendent aussi apparent le fait que le beau-fils redouble le personnage du metteur en scène. L'interprétation est renforcée par des échos énonciatifs : « Étonnante! Vous êtes étonnante! 》 $(P J: 47)$, s'écrie le metteur en scène lors d'une répétition d'Oh les beaux jours. « Vous êtes merveilleuse » (PJ : 53), s'exclame Raphaël, lorsque Flora joue pour lui le rôle de l'une des femmes historiques (Barbe Abbadie). Ou encore : «Vous êtes extraordinaire », après la première d'Oh les beaux jours (PJ : 188). 
roman, ${ }^{13}$ et suggère que Le Premier Jardin serait « le seul roman d'Anne Hébert où une jeune fille décide de mener la vie qu'elle souhaite en ne se laissant pas dévorer par sa mère ni par les règles sociales. » (Fonteneau, 2000 : 140) Sans pour autant nier le déploiement dans le roman d'une critique des structures sociales et représentatives patriarcales, assortie d'une célébration de la fertilité de la femme, il faudrait aussi prendre en compte certaines ambivalences. Comme le montre la présente analyse, I'oscillation des rôles féminins et l'effacement de la femme ne se placent pas uniquement sous le signe de la critique ni de l'agentivité au féminin. Le dénouement du roman est d'ailleurs extrêmement ambigu, et il invite à une lecture féministe plus nuancée. Car Raphaël joue non seulement le rôle d'un enfant, d'un ange, mais aussi celui d'un homme aux « dents de loup » $(P J: 82)$, à I'« allure féline » (PJ: 182), qui suit Maud à travers la ville jusqu'à une boîte de nuit, à la fin du récit ( $P J: 181-184)$, et auquel cette dernière aimerait d'abord « échapper». ( $P J: 182)$ Le chasseur et la proie sont des figures hébertiennes récurrentes, dont l'exemple le plus représentatif est celui de Stevens Brown des Fous de Bassan. Or, que le désir de la femme naisse à la fin du Premier Jardin - ou «transperc[e] », plutôt ( $P J$ : 184) -, il n'y a aucun doute : après un moment d'indécision dans le bar, Maud s'approche de Raphaël, et le couple danse désormais « en cadence ». ( $P J: 184)$ Mais il ne faudrait toutefois pas oublier le contexte malsain dans lequel se produit cette renaissance du désir, cette réconciliation du couple.

À la fin du Premier Jardin, « [I]a séparation a déjà eu lieu et l'exil où [Flora] est entrée la suit. » $(P J: 189)^{14}$ Exil de la mère, exil de la fille. Mais asile également, comme c'est le cas dans la pièce de Pirandello : rappelons que le point crucial de Chacun sa vérité est la question de savoir quelle femme fut enfermée, et à quel moment. Vu selon cette perspective, l'exil de Maud représenterait-il un refuge, ou un emprisonnement? La réponse à cette question ne se laisse pas trouver facilement. En effet, l'exil féminin semble constituer encore un autre de ces « points de mire des énigmes » que repère Marilyn Randall dans d'autres dénouements romanesques hébertiens (Randall, 1996 : 23).

13. Fonteneau retient ici le terme avancé par Lori Saint-Martin. À l'encontre du « postféminisme », « le métaféminisme n'annonce [pas] le déclin du féminisme; plutôt, il l'accompagne et l'enveloppe. Le préfixe signifie aussi "transformation", "participation", comme dans "métamorphose"; sens heureux pour le féminisme, qui a toujours revendiqué l'ouverture au changement, aux voix nouvelles. Ainsi, les écrits métaféministes affirment autant leur enracinement que leur différence, suggèrent à la fois qu'ils vont plus loin et qu'ils accompagnent, écoutent, tendent la main. » (Saint-Martin, 1992: 83)

14. Neil Bishop remarque que cette phrase constitue une référence intertextuelle à Claudel (Bishop, 1993 : 238). 
En d'autres mots, si l'on cherche une « clôture » à la question féministe (Randall, 1989), elle se trouve dans le champ de l'ambivalence. La matrice de la création au féminin se nourrit d'un discours patriarcal qui risque à tout moment de nuire au personnage féminin. Flora revendique des droits maternels et esthétiques, mais ses fantasmes filiaux témoignent de la prégnance de l'économie patriarcale qui façonne l'imaginaire et dont elle essaie de se libérer. Le texte laisse entendre, d'ailleurs, que la mère ne parviendra pas à protéger sa fille contre cette économie et ses schémas fantasmatiques, et que la création continuera d'avoir lieu au sein d'un contexte néfaste pour les femmes.

Flora se transforme en médium face aux personnages historiques et théâtraux qui sont morts, ou qui sont comme des morts-vivants (telles Winnie, ou Mme Frola de Chacun sa vérité de Luigi Pirandello). Pour elle, les femmes de I'Histoire québécoise et les personnages théâtraux qu'elle joue semblent exister sur le même continuum genré qui rappelle son passé personnel, et qui influence sa vie présente; ainsi, la « réalité » et la fiction s'enchevêtrent et se nourrissent. Notre analyse a démontré en particulier l'importance des personnages intertextuels de Winnie et de Mme Frola dans la revendication créative au féminin qu'entreprend Flora. Comme le note la critique beckettienne féministe, le mamelon de terre dans lequel Winnie se trouve ensevelie est un symbole de l'emprisonnement et de l'étouffement de la femme dans une idéologie patriarcale objectifiante et meurtrière selon laquelle la femme serait strictement définie par sa féminité. La position de Flora à l'égard de cet ensevelissement est problématique : bien que son interprétation mette en lumière les dangers de l'endossement d'un tel rôle essentialisant dans la vie « réelle », elle semble incapable de s'en dépouiller une fois hors scène. C'est, à tout le moins, ce que suggère le dénouement du roman, qui laisse entendre que Flora jouera le rôle de Mme Frola dans Chacun sa vérité, personnage qui disparaît derrière un rideau de passivité, qui fait la morte, selon la volonté du beau-fils. Nous avons mis en relief les similarités existantes entre la relation de Mme Frola avec son beau-fils et celle qu'entretient Flora avec Raphaël, soulignant le problème de l'acquiescement du personnage féminin au désir masculin.

Flora disparaît derrière des femmes disparues pour mieux les faire parler; I'ironie de ses efforts réside dans le fait qu'elle semble incapable d'échapper aux contraintes genrées qu'elle (dés)incarne et déconstruit, et dont elle est fort consciente. Le roman semble ainsi suggérer que toute appropriation de la scène historique ou théâ- 
trale patriarcale est inéluctablement périlleuse. Il présente néanmoins une fascinante imbrication de certains rôles (féminins) qu'ont joués les femmes au cours de l'Histoire, rôles sociaux, mais aussi esthétiques. Dans Le premier jardin, les femmes se trouvent au premier plan, et « la sainte lumière » de la scène romanesque illumine les expériences de celles-ci dans toute leur complexité. 


\section{Bibliographie}

ANCRENAT, Anne (2002), De mémoire de femmes : La " mémoire archaïque » dans l'œuvre romanesque d’Anne Hébert, Québec, Nota bene, coll. « Littérature(s) ».

BATTERSBY, Christine (1989), Gender and Genius: Towards a Feminist Aesthetics, Londres, The Women's Press.

BECKETT, Samuel (1974), Oh les beaux jours suivi de Pas moi, Paris, Minuit.

BENSTOCK, Shari (1990), « The Transformational Grammar of Gender in Beckett's Dramas », dans Linda Ben-Zvi (dir.), Women in Beckett: Performance and Critical Perspectives, Chicago, University of Illinois Press : $172-186$.

BEN-ZVI, Linda (dir.) (1990), «Introduction », dans Linda Ben-Zvi (dir.), Women in Beckett: Performance and Critical Perspectives, Chicago, University of Illinois Press : iv-xviii.

BISHOP, Neil (1993), Anne Hébert, son œuvre, leurs exils, Talence, Presses Universitaires de Bordeaux. BOUCHER-MARCHAND, Monique (2000), « La réécriture mythique du Premier jardin : une épiphanie moderne », Les Cahiers Anne Hébert, $\mathrm{n}^{\circ} 2$ : 43-57.

FONTENEAU, Anne (2000), « Le métaféminisme dans Le premier jardin d'Anne Hébert », Les Cahiers Anne Hébert, $\mathrm{n}^{\circ} 2$ : 127-144.

HÉBERT, Anne (1982), Les fous de Bassan, Paris, Seuil

HÉBERT, Anne (1988), Le premier jardin, Paris, Seuil.

HÉBERT, Anne (1993), CEuvre poétique 1950-1990, Montréal, Boréal, coll. « Boréal compact », $n^{\circ} 40$.

MITCHELL, Constantina et Paul R. CÔTÉ (1991), « Ordre et rite : la fonction du cortège dans Le premier jardin d'Anne Hébert », The French Review, vol. 64, n 3 : 451-462.

PERLOFF, Carey (1994), "Three Women and a Mound: Directing Happy Days », dans Lois Oppenheim (dir.), Directing Beckett, Ann Arbor, University of Michigan Press, coll. " Theatre: Theory/Text/Performance ».

PIRANDELLO, Luigi (1964) [1921], Théâtre I : Six personnages en quête d'auteur, Chacun sa vérité, Henri IV, Comme ci ou comme ça, trad. Benjamin Cremieux, Paris, Gallimard.

RANDALL, Marylin (1996), « Configurations rétrospectives dans l'œuvre d'Anne Hébert : L'enfant chargé de songes et la fin heureuse ", dans Michael Bishop (dir.), Thirty Voices in the Feminine, Amsterdam, Rodopi, coll. « Faux titre », $\mathrm{n}^{\circ} 114: 23-33$.

RANDALL, Marylin (1989), «Les énigmes des Fous de Bassan : féminisme, narration et clôture », Voix et images, vol. $15, \mathrm{n}^{\circ} 1(43)$ : 66-82.

ROY, Lucille (2006), « Anne Hébert ou le désert du monde », dans Janet M. Paterson et Lori Saint-Martin (dir.), Anne Hébert en revue, Québec/Montréal, Presses de l'Université du Québec/Voix et images : 51-70.

SAINT-MARTIN, Lori (1992), "Le métaféminisme et la nouvelle prose féminine au Québec », Voix et images, vol. 18, $\mathrm{n}^{\circ} 1: 78-88$.

SAINT-MARTIN, Lori (2006), « Les premières mères, Le premier jardin », dans Janet M. Paterson et Lori Saint-Martin (dir.), Anne Hébert en revue, Québec/Montréal, Presses de l'Université du Québec/Voix et images : 207-218. 